\title{
Nongenetic Risk Factors for Neovascular Age-Related Macular Degeneration
}

\author{
Tina Ristau, ${ }^{1}$ Lebriz Ersoy, ${ }^{1}$ Moritz Hahn, ${ }^{2}$ Anneke I. den Hollander, ${ }^{3}$ Bernd Kirchhof, ${ }^{1}$ \\ Sandra Liakopoulos, ${ }^{1}$ and Sascha Fauser ${ }^{1}$ \\ ${ }^{1}$ Department of Ophthalmology, University Hospital of Cologne, Cologne, Germany \\ ${ }^{2}$ Institute of Medical Statistics, Informatics and Epidemiology, University of Cologne, Cologne, Germany \\ ${ }^{3}$ Department of Ophthalmology, Radboud University Nijmegen Medical Center, Nijmegen, The Netherlands
}

Correspondence: Sascha Fauser, Department of Ophthalmology, University Hospital of Cologne, Kerpener Str. 62, 50924 Cologne, Germany; sascha.fauser@uk-koeln.de.

TR and LE contributed equally to the work presented here and therefore should be regarded as equivalent authors.

Submitted: March 4, 2014

Accepted: July 13, 2014

Citation: Ristau T, Ersoy L, Hahn M, et al. Nongenetic risk factors for neovascular age-related macular degeneration. Invest Ophthalmol Vis Sci. 2014;55:5228-5232. DOI:10.1167/ iovs.14-14299
Purpose. To create a risk model for neovascular age-related macular degeneration (nAMD) based on nongenetic factors.

Methods. In this case-control study, 1459 individuals were included, 445 patients showed nAMD and 1014 were healthy controls. Participants were randomly assigned into a training set (containing two-thirds of individuals) and a validation set. Stepwise logistic regression analysis was performed for 25 environmental risk factors in the training set. The risk model with the remaining factors was then validated in the validation set using receiver operating characteristics (ROC) curve and Hosmer-Lemeshow-Test. Additionally, a genetic risk model including variants in the complement factor $\mathrm{H}$ gene (CFH, rs1061170) and the age-related maculopathy susceptibility 2 gene (ARMS2, rs10490924) was generated.

REsults. The environmental risk model with the factors age, alcohol use, allergy, education, sunlight exposure, fish consumption, and physical exercise showed an AUC of 0.80 (95\% confidence interval [CI] 0.76-0.84) in the training set. Validation of the model showed adequate calibration (Hosmer-Lemeshow $P=0.81$ ). The AUC for the genetic model was 0.77 (95\% CI 0.730-0.808), for the combined environmental and genetic model 0.92 (95\% CI $0.887-0.947)$.

Conclusions. Seven nongenetic factors are able to provide equivalent discrimination between nAMD patients and controls to genetic risk models. Most of them are modifiable and give the opportunity for counseling patients.

Keywords: age-related macular degeneration, nongenetic risk factor

\begin{abstract}
A ge-related macular degeneration (AMD), especially the late forms with geographic atrophy of the retinal pigment epithelium or choroidal neovascularization (CNV), is the leading cause of severe blindness of the elderly population in the developed world. The etiology of AMD is multifactorial, involving genetic and environmental factors. The two major loci for AMD development are variants in the complement factor $\mathrm{H}(\mathrm{CFH})$ gene and the age-related maculopathy susceptibility 2 (ARMS2) gene, which account for $70 \%$ of the genetic risk. $^{1,2}$
\end{abstract}

To predict the risk in complex diseases, analysis of single aspects is generally of limited value and evaluating multiple independent disease-associated factors may be more appropriate. ${ }^{3}$ This can be based on risk models. But so far, they were either based on exclusively genetic factors ${ }^{4-7}$ or a combination of genetic and few nongenetic factors. ${ }^{8-12}$

Although, besides age, the environmental factors of smoking, cardiovascular risk factors, diabetes, higher body mass index (BMI), nutrition, and lifestyle factors, such as sunlight exposure and alcohol consumption, may be associated with AMD ${ }^{13-22}$ an extensive risk model involving environmental factors has not been established.

In our study, we analyzed 25 nongenetic variables and developed a risk model for nAMD. Furthermore, we tested the model with the addition of major genetic polymorphisms.

\section{MeTHODS}

From the European Genetic Database (EUGENDA, www. eugenda.org), clinical, genetic, and imaging data of 1878 individuals were reviewed. Patients and controls were recruited from a small area around Cologne, in Western Germany. Recruitment was initiated by participants and was not "hospital-based." The study was performed in accordance with the tenets of the Declaration of Helsinki and the Medical Research Involving Human Subjects Act (WMO) and was approved by the local ethics committee. Written informed consent was obtained from all participants.

Patient data included age (subdivided into 8 groups: 50-60 years, 61-65 years, 66-70 years, $71-75$ years, $76-80$ years, $81-$ 85 years, 86-90 years, and $>90$ years), sex (female/male), BMI (subdivided in three groups: normal: $<25$, overweight 2529.99 and obese $\geq 30$ ), marital status (living alone/living together), highest education level (no school degree or elementary school, high school, professional education, university degree), and iris color (light [blue, gray], medium [green, hazel], dark [brown]). Medical history was obtained by an interviewer-assisted questionnaire and included arterial hypertension, myocardial infarction, angina pectoris, stroke or transient ischemic attack (TIA), congestive heart failure, vascular bypass surgery, other heart disease, blood-clotting 


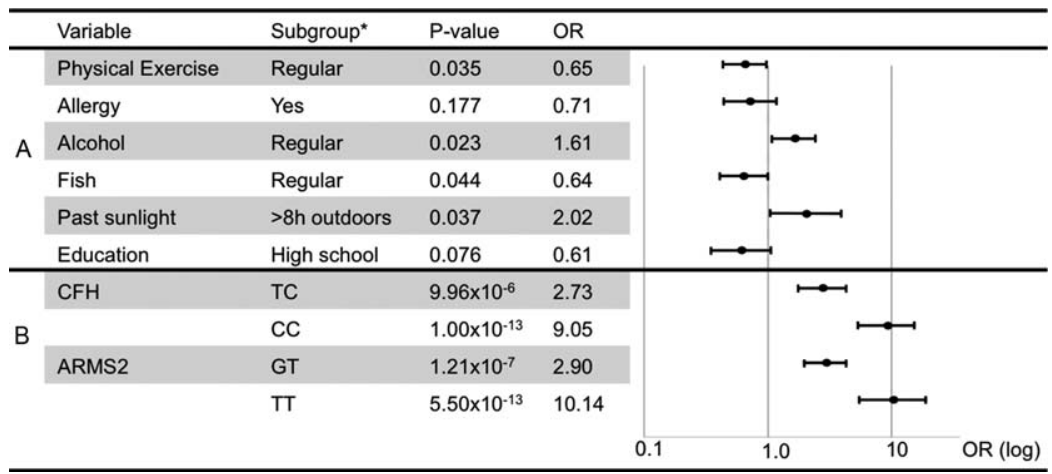

Figure 1. Multivariate logistic regression analysis for the environmental risk model (A) and ARMS2 and CFH (B) displaying ORs and 95\% CIs. *As reference served, no physical exercise, negative history of allergy, nonregular alcohol use, no fish intake, past sunlight exposure of less than 8 hours daily, and no/elementary school degree (high school displayed exemplarily; OR for professional education 0.59 and for university 0.68 ).

disorder, diabetes, rheumatoid arthritis, thyroid disease, cancer, liver disease, kidney disease, lung disease, migraine, and history of allergy (including all types of allergies, but primarily pollen allergy and house dust mite allergy). Furthermore, data about the use of platelet inhibitors, nonsteroidal anti-inflammatory drugs (NSAIDs), antihypertensive drugs, antidiabetic drugs, corticosteroids, coumarin derivates, and antiglaucomatous drugs was evaluated (yes/no). Data of the lifestyle factors smoking (never/ever); regular alcohol use (yes/no); regular (at least once a week) intake of fruit, vegetables, fish, red meat, and physical exercise; and past sunlight exposure (more or less than 8 hours outside activity per day during working life).

For a validated environmental risk model, the dataset was randomly subdivided into a training set, containing two-thirds of cases and a validation set containing one-third of cases.

All cases were included that either were controls or had neovascular AMD (nAMD). Staging of AMD was performed by grading of retinal images, including stereo color fundus photographs (FPs) and, if available, fluorescein angiograms (FAs, available in $40 \%$ of cases) and spectral domain optical coherence tomograms (SDOCTs, available in $81 \%$ of cases), according to the standard protocol of the Cologne Image Reading Center by certified graders (TR, LE). Patients without drusen and patients having only small drusen $(<63 \mu \mathrm{m})$ or pigmentary changes and fewer than 10 small drusen were defined as controls. Neovascular AMD was classified as CNV secondary to AMD in at least one eye. Choroidal neovascularization was termed as choroidal neovascular lesion within the Early Treatment Diabetic Retinopathy Study grid on FPs, Fas, or SDOCTs, when there was evidence for fluid, blood, or fibrovascular tissue on FPs, active classic or occult CNV, or signs for previous CNV, such as staining scar on FAs and/or retinal or subRPE fluid and/or tissue on SDOCT.

\section{Genotyping}

Genotyping of single nucleotide polymorphisms in the $\mathrm{CFH}$ (Y402H; rs1061170) and ARMS2 (A69S; rs10490924) genes was performed with TaqMan probes and primers from Applied Biosystems (Foster City, CA, USA). The assays were analyzed on the ABI 7900HT system (Applied Biosystems) according to the protocols provided by the manufacturer.

\section{Statistical Analysis}

All calculations were performed using SPSS software version 21.0 (IBM Software and Systems, Armonk, NY, USA). Risk factors with less than $5 \%$ of answers per response option were excluded. In the training data set, an environmental risk model for nAMD was created using stepwise logistic regression analysis with all environmental risk factors. The model was validated using the Hosmer-Lemeshow test with the equation

$$
\mathrm{T}=\sum_{i=1}^{k}\left(\left(O_{i}-E_{i}\right)^{2} /\left(E_{i} x E_{i} / n_{i}\right)\right) \sim \chi_{k-2}^{2}
$$

to check the calibration and the receiver operating characteristic (ROC) curve and the area under the curve (AUC) to check the discrimination ability of the model. In addition, the risk model was applied to the validation set and the HosmerLemeshow test and the ROC curve and AUC were calculated.

Associations between CFH and ARMS2 and nAMD were evaluated in the training set by logistic regression analysis. Both genetic risk factors were combined with the variables of the environmental risk model and a combined model was calculated using multivariate logistic regression analysis. Accordingly, the Hosmer-Lemeshow test, the ROC curve, and the AUC for the combined model was determined.

\section{Results}

The training set contained 291 (29.8\%) patients with nAMD and $685(70.2 \%)$ healthy controls; the validation set included 154 (31.9\%) nAMD cases and 329 (68.1\%) controls. Mean age of nAMD patients in the training set was $77.6 \pm 8.4$ years and $70.2 \pm 7.6$ years for controls, 575 (58.9\%) were female. In the validation set, mean age was $77.1 \pm 8.2$ years for nAMD cases and $70.0 \pm 7.7$ for controls and 285 were female (59.0\%).

The variables angina pectoris, stroke or TIA, congestive heart failure, blood-clotting disorder, vascular bypass surgery, liver disease, NSAIDs, corticosteroids, coumarin derivates, and fruit and vegetable intake were excluded from analysis because less than $5 \%$ of answers were given for one response option.

\section{Creation and Validation of Environmental Risk Model}

Stepwise regression analysis was performed by computing the factors age, regular fish consumption, allergy, regular alcohol use, physical exercise, sunlight exposure, and education level as relevant factors associated with the risk of nAMD (Fig. 1). The model seemed to be well calibrated (Hosmer-Lemeshow test: $P=0.42$ ) and was able to discriminate between cases and controls quite well (AUC 0.80, 95\% confidence interval [CI] 0.76-0.84) (Fig. 2). The risk model was further validated in the validation set. Calibration and discrimination was similar to the 


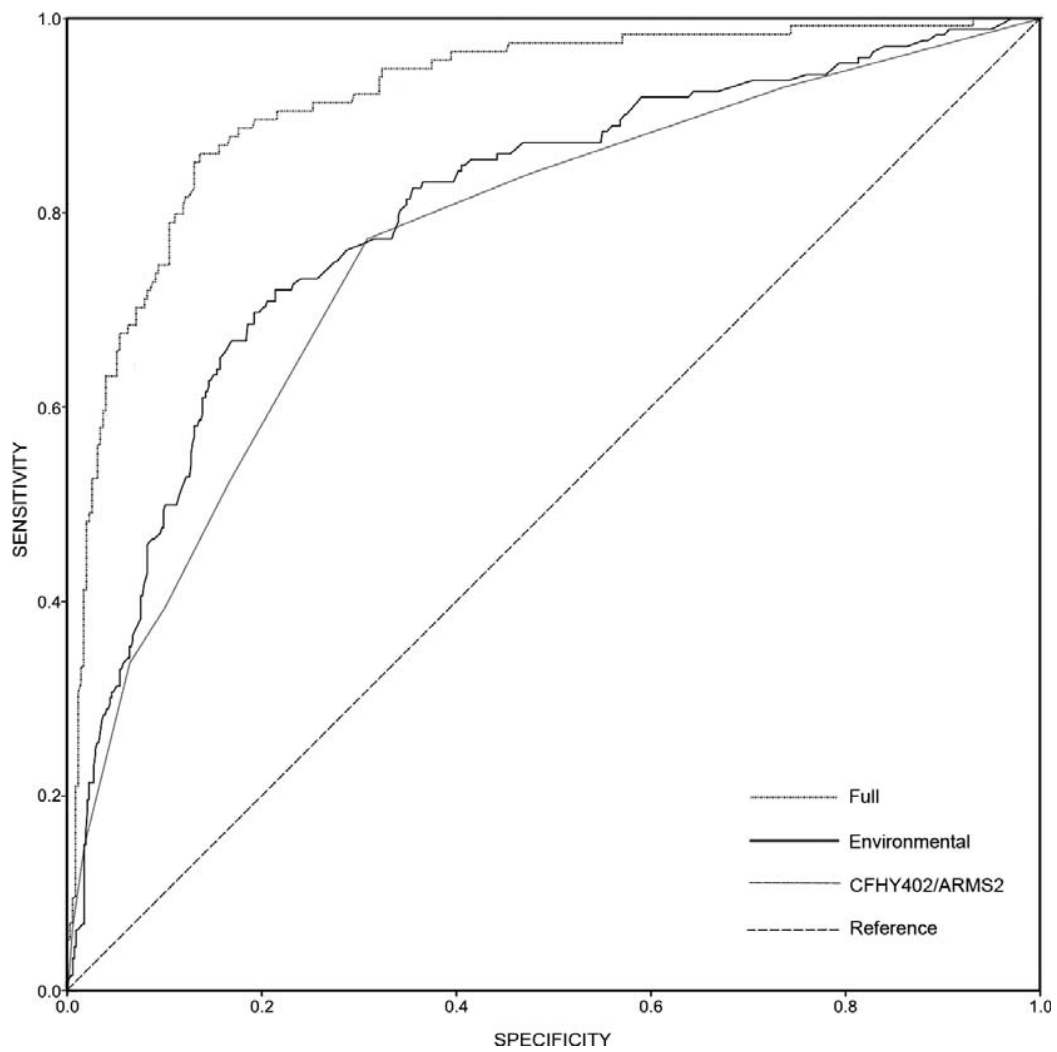

Figure 2. The ROCs for the discrimination between nAMD and no AMD showed an AUC of 0.80 (95\% CI $0.764-0.841$ ) for the environmental model, 0.77 (95\% CI 0.730-0.808) for the genetic model, and 0.92 (95\% CI 0.887-0.947) for the full model.

values in the training set (Hosmer-Lemeshow test: $P=0.81$; AUC 0.82, 95\% CI 0.77-0.87; Fig. 3).

\section{Implication of ARMS2 and CFH in the Environmental Risk Model}

Genetic information was available for ARMS2 in 639 cases (65.4\%) and for $\mathrm{CFH}$ in 637 cases (65.2\%) in the training set.

Logistic regression analysis of genetic risk factors showed an association for ARMS2 (for heterozygous risk allele: odds ratio $[\mathrm{OR}] 2.90,95 \% \mathrm{CI} 1.96-4.31, P=1.21 \times 10^{-7}$; for homozygous risk allele: $\mathrm{OR} 10.14,95 \% \mathrm{CI}$ 5.42-18.99, $P=1.01$ $\times 10^{-13}$ ) and CFH (for heterozygous risk allele: OR $2.73,95 \% \mathrm{CI}$ 1.75-4.26, $P=9.96 \times 10^{-6}$; for homozygous risk allele: OR 9.05, 95\% CI 5.38-15.23, $P=1.00 \times 10^{-13}$ ) with nAMD (Fig. 1). The ROC curve is displayed in Figure 2 and showed an AUC of 0.77 (95\% CI 0.730-0.808; Hosmer-Lemeshow test: $P=0.17$; Fig. 2).

The ROC of environmental and genetic risk factors in a combined model (full model) resulted in an AUC of 0.92 (95\% CI 0.887-0.947; Hosmer-Lemeshow test: $P=0.71$; Fig. 2)

\section{Discussion}

Although risk models for AMD based on genetic polymorphisms are well established, less is known for nongenetic factors. Therefore, we developed a nongenetic risk model for nAMD by evaluating 25 factors. Seven variables allowed a high discrimination (AUC 0.80) between controls and nAMD patients and adequate validation in a separate replication cohort indicates that our model would presumably perform well in other populations.
The genetic background, especially variants in ARMS2 and CFH genes are important for AMD development and various genetic risk models exist. ${ }^{4-7}$ Our genetic model based on two polymorphisms in ARMS2 and CFH showed comparable discrimination (AUC 0.77 ) to the model created by Jakobsdottir et al $^{6}$ containing three SNPs (0.79), however not reaching the AUC values of our nongenetic model. Even genetic models

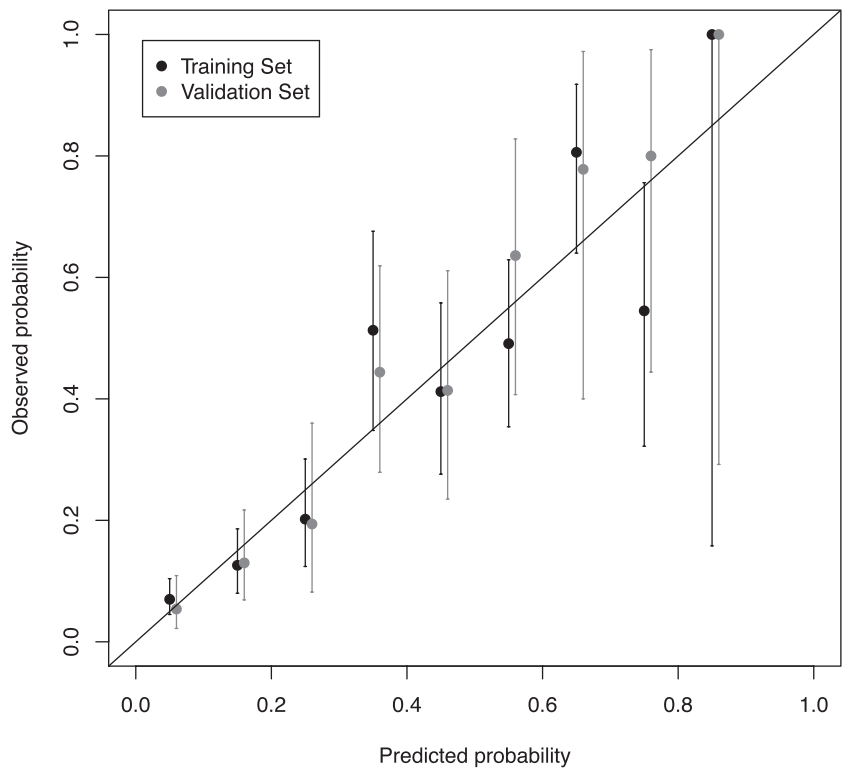

FIGURE 3. Comparison of risk deciles in the training set and the validation set for the environmental risk model. 
consisting of up to 19 SNPs could not approach better discrimination between AMD cases and controls, $, 4,5,7$ indicating that even highly associated SNPs may not always be effective as classifiers ${ }^{6}$ and underlining the crucial role of the environmental factors. The addition of only two genetic polymorphisms increased the AUC to 0.92, which is higher than in any other cross-sectional study combining environmental and genetic factors. ${ }^{8,10,12}$ Other risk models included genetic factors and systemic biomarkers ${ }^{9,11}$; however, they also revealed lower discrimination than our combined model. Only in longitudinal progression models did AUC also reach levels above 0.90 depending on the inclusion of phenotypic baseline characteristics. ${ }^{19,23-25}$

Of 25 factors that were analyzed, 7 factors were identified as important for discrimination: age, alcohol use, allergy, fish consumption, education, sunlight exposure, and physical exercise.

In our cohort, regular alcohol use was associated with nAMD. Our results are in line with previous reports. ${ }^{26-28}$ Alcohol is a drug that could be avoided to lower the risk for AMD development.

A positive history of allergy was found to have a protective effect for nAMD in our cohort, which was also found in another study. ${ }^{29}$ Literature regarding allergy and AMD is limited and the relationship between these two common conditions is not yet understood and should be investigated in further studies.

The protective effect of a higher education level for AMD development goes in line with previous reports. ${ }^{15,30}$ The explanation for the effect may be difficult. Different education levels may come along with other behavioral and lifestyle factors, which are not identified yet.

Regular physical exercise is known to be protective for various diseases, for example coronary heart disease and type 2 diabetes. $^{31,32}$ The Beaver Dam Eye Study has shown that patients with an active lifestyle were less likely to develop nAMD. ${ }^{33}$ In our cohort, nAMD appeared significantly less frequently in patients with weekly physical exercise, revealing that constant physical activity may be effective for AMD prevention.

Sunlight exposure has been shown to be a risk factor for AMD in epidemiological studies. ${ }^{22}$ This finding could also be confirmed in our cohort. Protection against sunlight may also help to lower the risk of developing nAMD.

Previous reports could show the protective effects of high fish intake. ${ }^{34,35}$ In our study, regular fish intake also was a protective factor against nAMD and can be recommended.

Many of the ascertained nongenetic factors in our model could be modified to eventually reduce the occurrence of nAMD and patients may contribute actively to reduce the risk for CNV by omitting alcohol and following an active lifestyle. Our data demonstrate that a model based on factors that can be fast and easily obtained by a few questions has a high accuracy and involves obviously less cost than any genetic analysis.

Our study was well powered, validated in a separate sample, and included a high number of nAMD patients. To our knowledge, this is the first study to analyze most of the currently known nongenetic factors developing a risk model to predict nAMD.

Additionally, multimodal imaging for AMD staging in EUGENDA reduced misclassification of AMD phenotypes. Limitations of our study are the use of questionnaires for evaluation of medical history. It might be assumed to be a limitation but also a strength that our study included exclusively nAMD cases with CNV in at least one eye, as well as controls with no signs of early or late AMD in any eye. The neovascular form of AMD is important for patients, causing significant visual impairment. Additionally, the possibility of interactions among the environmental factors (e.g., a coincidence of high alcohol use and low fish intake or higher education and a possibly more active lifestyle) cannot be excluded, but the composition of a stepwise regression model ensures that each included risk factor has an additional essential effect on the model.

In summary, our analysis revealed the important role of a few environmental factors that allow accurate discrimination between controls and nAMD cases that are equivalent to genetic risk models.

\section{Acknowledgments}

Disclosure: T. Ristau, None; L. Ersoy, None; M. Hahn, None; A.I. den Hollander, None; B. Kirchhof, None; S. Liakopoulos, None; S. Fauser, None

\section{References}

1. Klein RJ, Zeiss C, Chew EY, et al. Complement factor $\mathrm{H}$ polymorphism in age-related macular degeneration. Science. 2005;308:385-389.

2. Jakobsdottir J, Conley YP, Weeks DE, Mah TS, Ferrell RE, Gorin MB. Susceptibility genes for age-related maculopathy on chromosome 10q26. Am J Hum Genet. 2005;77:389-407.

3. Janssens AC, Aulchenko YS, Elefante S, Borsboom GJ, Steyerberg EW, van Duijn CM. Predictive testing for complex diseases using multiple genes: fact or fiction? Genet Med. 2006;8:395-400.

4. Fritsche LG, Chen W, Schu M, et al. Seven new loci associated with age-related macular degeneration. Nat Genet. 2013;45: 433-439, 439e1-2.

5. Grassmann F, Fritsche LG, Keilhauer CN, Heid IM, Weber BH. Modelling the genetic risk in age-related macular degeneration. PLoS One. 2012;7:e37979.

6. Jakobsdottir J, Gorin MB, Conley YP, Ferrell RE, Weeks DE. Interpretation of genetic association studies: markers with replicated highly significant odds ratios may be poor classifiers. PLoS Genet. 2009;5:e1000337.

7. Hageman GS, Gehrs K, Lejnine S, et al. Clinical validation of a genetic model to estimate the risk of developing choroidal neovascular age-related macular degeneration. Hum Genomics. 2011;5:420-440.

8. Farwick A, Dasch B, Weber BH, Pauleikhoff D, Stoll M, Hense HW. Variations in five genes and the severity of age-related macular degeneration: results from the Muenster aging and retina study. Eye (Lond). 2009;23:2238-2244.

9. Hecker LA, Edwards AO, Ryu E, et al. Genetic control of the alternative pathway of complement in humans and age-related macular degeneration. Hum Mol Genet. 2010;19:209-215.

10. McKay GJ, Dasari S, Patterson CC, Chakravarthy U, Silvestri G. Complement component 3: an assessment of association with AMD and analysis of gene-gene and gene-environment interactions in a Northern Irish cohort. Mol Vis. 2010;16: 194-199.

11. Scholl HP, Charbel Issa $P$, Walier M, et al. Systemic complement activation in age-related macular degeneration. PLOS One. 2008;3:e2593.

12. Spencer KL, Olson LM, Schnetz-Boutaud N, et al. Using genetic variation and environmental risk factor data to identify individuals at high risk for age-related macular degeneration. PLoS One. 2011;6:e17784.

13. Tan JS, Mitchell P, Smith W, Wang JJ. Cardiovascular risk factors and the long-term incidence of age-related macular degeneration: the Blue Mountains Eye Study. Ophthalmology. 2007; 114:1143-1150.

14. Chakravarthy U, Augood C, Bentham GC, et al. Cigarette smoking and age-related macular degeneration in the EUREYE Study. Ophthalmology. 2007;114:1157-1163. 
15. Age-Related Eye Disease Study Research Group. Risk factors associated with age-related macular degeneration. A casecontrol study in the age-related eye disease study. Age-Related Eye Disease Study Report Number 3. Ophthalmology 2000; 107:2224-2232.

16. Sperduto RD, Hiller R. Systemic hypertension and age-related maculopathy in the Framingham Study. Arch Ophthalmol. 1986;104:216-219.

17. Hyman L, Schachat AP, He Q, Leske MC. Hypertension, cardiovascular disease, and age-related macular degeneration. Age-Related Macular Degeneration Risk Factors Study Group. Arch Ophthalmol. 2000;118:351-358.

18. Klein R, Klein BE, Moss SE. Diabetes, hyperglycemia, and agerelated maculopathy. The Beaver Dam Eye Study. Ophthalmology. 1992;99:1527-1534.

19. Seddon JM, Cote J, Davis N, Rosner B. Progression of agerelated macular degeneration: association with body mass index, waist circumference, and waist-hip ratio. Arch $O p h$ thalmol. 2003;121:785-792.

20. Cho E, Hung S, Willett WC, et al. Prospective study of dietary fat and the risk of age-related macular degeneration. Am J Clin Nutr. 2001;73:209-218.

21. Chong EW, Kreis AJ, Wong TY, Simpson JA, Guymer RH. Alcohol consumption and the risk of age-related macular degeneration: a systematic review and meta-analysis. $\mathrm{Am} \mathrm{J}$ Ophthalmol. 2008;145:707-715.

22. Sui GY, Liu GC, Liu GY, et al. Is sunlight exposure a risk factor for age-related macular degeneration? A systematic review and meta-analysis. Br J Ophthalmol. 2013;97:389-394.

23. Seddon JM, Reynolds R, Yu Y, Daly MJ, Rosner B. Risk models for progression to advanced age-related macular degeneration using demographic, environmental, genetic, and ocular factors. Ophthalmology. 2011;118:2203-2211.

24. Seddon JM, Reynolds R, Yu Y, Rosner B. Validation of a prediction algorithm for progression to advanced macular degeneration subtypes. JAMA Ophthalmol. 2013;131:448455 .
25. Klein R, Myers CE, Meuer SM, et al. Risk alleles in CFH and ARMS2 and the long-term natural history of age-related macular degeneration: the Beaver Dam Eye Study. JAMA Ophthalmol. 2013;131:383-392.

26. Knudtson MD, Klein R, Klein BE. Alcohol consumption and the 15-year cumulative incidence of age-related macular degeneration. Am J Ophthalmol. 2007;143:1026-1029.

27. Buch H, Vinding T, la Cour M, Jensen GB, Prause JU, Nielsen NV. Risk factors for age-related maculopathy in a 14-year follow-up study: the Copenhagen City Eye Study. Acta Ophthalmol Scand. 2005;83:409-418.

28. Cho E, Hankinson SE, Willett WC, et al. Prospective study of alcohol consumption and the risk of age-related macular degeneration. Arch Ophthalmol. 2000;118:681-688.

29. Ristau T, Ersoy L, Lechanteur Y, et al. Allergy is a protective factor against age-related macular degeneration. Invest $O p h$ thalmol Vis Sci. 2014;55:210-214.

30. Seddon JM, Reynolds R, Maller J, Fagerness JA, Daly MJ, Rosner B. Prediction model for prevalence and incidence of advanced age-related macular degeneration based on genetic, demographic, and environmental variables. Invest Ophthalmol Vis Sci. 2009;50:2044-2053.

31. Morris JN. Exercise in the prevention of coronary heart disease: today's best buy in public health. Med Sci Sports Exerc. 1994;26:807-814.

32. Gill JM, Cooper AR. Physical activity and prevention of type 2 diabetes mellitus. Sports Med. 2008;38:807-824.

33. Knudtson MD, Klein R, Klein BE. Physical activity and the 15year cumulative incidence of age-related macular degeneration: the Beaver Dam Eye Study. Br J Ophthalmol. 2006;90: 1461-1463.

34. Seddon JM, Cote J, Rosner B. Progression of age-related macular degeneration: association with dietary fat, transunsaturated fat, nuts, and fish intake. Arch Ophthalmol. 2003;121: 1728-1737.

35. Swenor BK, Bressler S, Caulfield L, West SK. The impact of fish and shellfish consumption on age-related macular degeneration. Ophthalmology. 2010;117:2395-2401. 